\title{
Spontaneous persistent currents in a quantum spin Hall insulator
}

\author{
D. Soriano ${ }^{1,2}$ and J. Fernández-Rossier ${ }^{1}$ \\ ${ }^{1}$ Departamento de Fìsica Aplicada, Universidad de Alicante, San Vicente del Raspeig, Spain \\ ${ }^{2}$ Instituto de Ciencia de Materiales de Madrid, CSIC, Cantoblanco, E-28049 Madrid, Spain
}

(Received 20 September 2010; published 7 October 2010)

\begin{abstract}
We present a mechanism for persistent charge current. Quantum spin Hall insulators hold dissipationless spin currents in their edges so that, for a given spin orientation, a net charge current flows which is exactly compensated by the counterflow of the opposite spin. Here we show that ferromagnetic order in the edge upgrades the spin currents into persistent charge currents without applied fields. For that matter, we study the Hubbard model including Haldane-Kane-Mele spin-orbit coupling in a zigzag ribbon and consider the case of graphene. We find three electronic phases with magnetic edges that carry currents reaching $0.4 \mathrm{nA}$, comparable to persistent currents in metallic rings, for the small spin-orbit coupling in graphene. One of the phases is a valley half metal.
\end{abstract}

DOI: 10.1103/PhysRevB.82.161302

PACS number(s): 72.80.Vp, 03.65.Vf, 73.23.Ra

Ordered electronic phases can emerge in condensed matter with properties fundamentally different from those of the constituent atoms. Two main different scenarios are known that result in the emergence of nontrivial electronic order. On one side, spontaneous symmetry breaking driven by manybody interactions which accounts for the existence of the crystalline order in solids and the variety of ordered electronic phases they can present, such as superconductivity and ferromagnetism. ${ }^{1}$ On the other side, topological order, which accounts for the robust quantized properties of the electron gas in the quantum Hall regimes, and, more recently, on the properties of the so-called topological insulators. ${ }^{2-5}$ Whereas integer quantum Hall state is driven by an external magnetic field, topological insulators are driven by spin-orbit (SO) interaction. They are different from conventional insulators because of their conducting surface (or edge) spin states which can be either chiral or spin filtered and are robust with respect to time-reversal symmetric perturbations. ${ }^{2-6}$

The experimental finding of topological insulators ${ }^{7,8}$ motivates the general questions of whether and how electronic interactions could produce electronic phase transitions in their surface (or edge) states and what would be the consequences of such symmetry breaking. Here we address these questions by including the effect of the interactions into the Haldane-Kane-Mele model for quantum spin Hall insulators ${ }^{3,4,9}$ defined in a honeycomb lattice stripe. Some approximate physical realizations of this model are cold fermionic atoms trapped in suitably engineered optical lattices, ${ }^{10}$ a modulated two-dimensional electron gas, ${ }^{11}$ the transition-metal oxide $\mathrm{Na}_{2} \mathrm{IrO}_{3},{ }^{12}$ and the most studied so far, graphene. The enormous interest in graphene ribbon with zigzag edges ${ }^{3,4,13-24}$ arises in the context of in the context of spintronics, ${ }^{3,4}$ magnetoelectronics, ${ }^{14,16,19,20,22,24}$ and valleytronics. ${ }^{18}$ When described with a one-orbital tightbinding model, graphene zigzag ribbons are conducting because of two degenerate almost flat bands associated to states localized at the edges. ${ }^{13}$ Importantly, the zigzag states preserve the valley character ${ }^{15,18}$ of two-dimensional graphene. When considered separately, the effect of electronic repulsion and spin-orbit interactions over the system is dramatic and has been widely studied. On one side, it was soon recognized that electron Coulomb repulsion, in the Hubbard model, results in ferromagnetic order at the edges of the ribbon. ${ }^{14}$ In the ground state the edges are counterpolarized and according to both density-functional calculations ${ }^{16}$ and to mean-field Hubbard model, ${ }^{22}$ a gap opens so that the ribbon is insulating.

When Coulomb repulsion is neglected, SO interaction opens a gap in the spectrum of bulk graphene ${ }^{25,26}$ which is accompanied by the emergence of spin-filtered edge states at the Fermi energy. ${ }^{3,4}$ These two features, a spin-orbit driven gap and the emergence of topologically robust edge states, are the hallmark of the quantum spin Hall insulator phase. ${ }^{2-4}$ Thus, the Coulomb driven and the SO driven phase have very different magnetic and conducting properties. Here we address the electronic properties of the ribbon when both Coulomb repulsion and spin-orbit coupling are considered within a mean-field Hubbard model ${ }^{14,19,22}$ with Kane-Mele spin-orbit coupling. ${ }^{3,4,9}$ We report two main findings. First, in the presence of SO coupling the states with counterpolarized ferromagnetic edges break valley symmetry and, above a critical SO strength, the gap closes in one valley only, resulting in a valley half metal (HM). Second, in the presence of SO coupling ferromagnetic edges give rise to charge currents in the edges without an applied magnetic field. We consider the one-orbital Hubbard model in a honeycomb zigzag ribbon, at half filling, with the addition of the Kane-Mele SO coupling, ${ }^{3}$

$$
\mathcal{H}=\sum_{i, j, \sigma} t_{i, j}(\sigma) c_{i, \sigma}^{\dagger} c_{j, \sigma}+U \sum_{i} n_{i \uparrow} n_{i \downarrow}
$$

The hopping matrix $t_{i j}(\sigma)$ in the first term accounts both for the standard first-neighbor spin-independent hopping $(t)$ and the Kane-Mele-Haldane second-neighbor spin-orbit coupling. $3,4,9$ The amplitude of the latter is given by the expression $i t_{K M} \sigma \hat{z} \cdot\left(\vec{d}_{1} \times \vec{d}_{2}\right)$, where $\vec{d}_{1,2}$ are unit vectors along the direction of the bond that connect sites $i$ and $j$ with their common first neighbor [see Fig. 1(a)], $\hat{z}$ is the unit vector normal to the ribbon plane, and $\sigma= \pm 1$ indexes the spin projection along $\hat{z}$. For a flat ribbon, the SO term commutes with $\sigma_{z}$. The second term in Eq. (1) describes Coulomb repulsion between electrons in the Hubbard approximation. We 

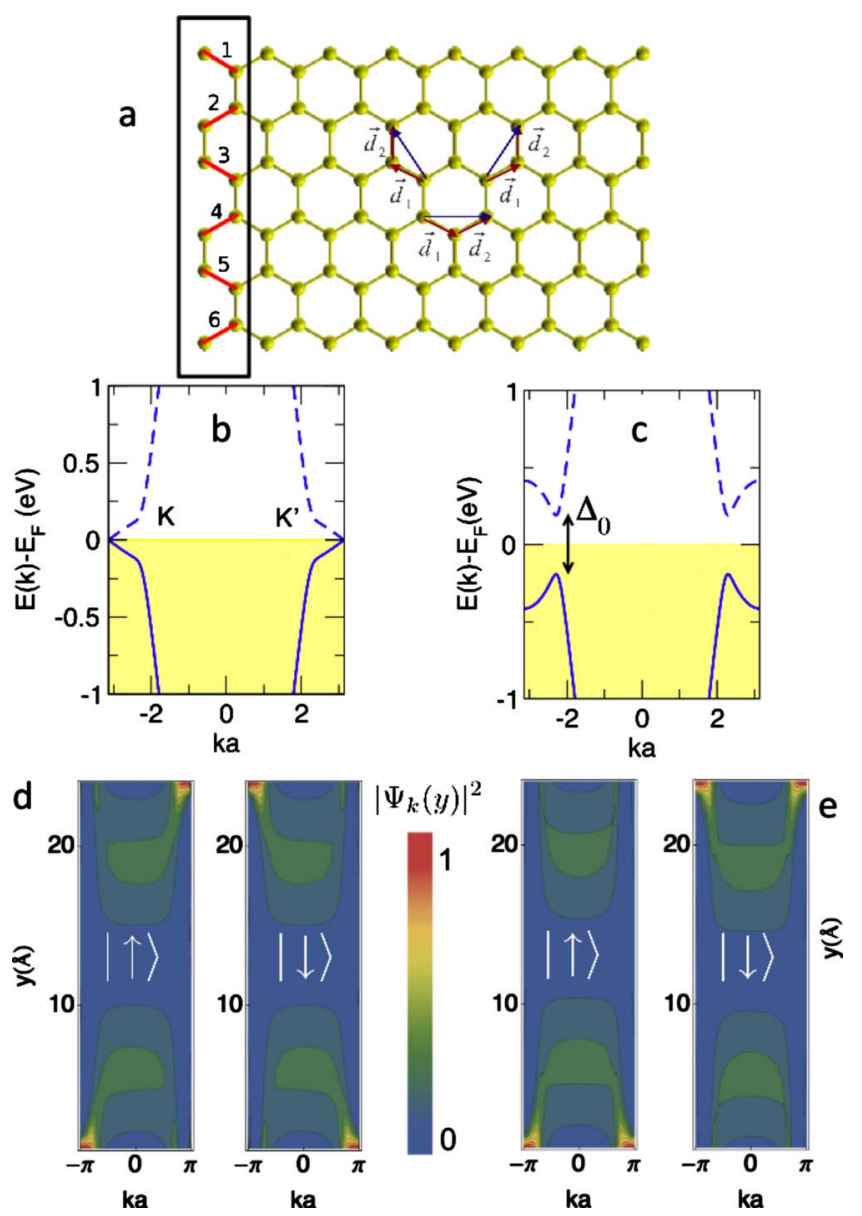

FIG. 1. (Color online) (Color online) (a) Honeycomb zigzag ribbon with $N_{y}=6$. The second-neighbor hopping vectors $\vec{d}_{i}$ are shown. (b) Low-energy bands for the spin Hall phase in $N_{y}=12$ ribbon $\left(U=0, t_{K M}=0.01 \mathrm{eV}\right)$. (c) Low-energy bands for the same ribbon with counterpolarized ferromagnetic edges $(U=3 \mathrm{eV}$ and $\left.t_{K M}=0\right)$. (d) Contour map of the valence-band wave function $\left|\Psi_{k \sigma \nu}(y)\right|^{2}$ for spin up (left panel) and spin down (right panel). (e) Same as (d) for the magnetic ribbon.

treat it in a mean-field approximation so that we end up with an effective single-particle Hamiltonian where the electrons interact with a spin-dependent potential that is calculated self-consistently, $U \Sigma_{i}\left(n_{i, \uparrow}\left\langle n_{i, \downarrow}\right\rangle+n_{i, \downarrow}\left\langle n_{i, \uparrow}\right\rangle\right)$, so that $\sigma_{z}$ is a good quantum number. Zigzag ribbons are defined by $N_{y}$, the number of zigzag chains which yield a total of $2 N_{y}$ atoms per unit cell in a one-dimensional crystal [Fig. 1(a)]. Importantly, the top and bottom edges belong to the two different triangular sublattices that define the honeycomb lattice. For a given wave number $k$ and spin $\sigma$ the mean-field Hamiltonian has $2 N_{y}$ states $\Psi_{k \sigma \nu}(y)$ with energy $\epsilon_{\sigma \nu}(k)$.

We consider first the model in the two limit cases, $U$ $=0, t_{K M}>0$ and $t_{K M}=0, U>0$ for a ribbon with $N_{Y}=12$. The highest occupied and lowest empty energy bands of the $U=0, t_{K M}>0$ case are shown in Fig. 1(b). The special spinfiltered edge states are the linear bands crossing the Fermi energy $\left(E_{F}\right)$. The wave function squared, $\left|\Psi_{k, \sigma, \nu}(y)\right|^{2}$, of the valence-band states are represented in Fig. 1(d) for $\sigma=\uparrow$ and $\sigma=\downarrow$, respectively. It is apparent that $\sigma=\uparrow(\sigma=\downarrow)$ electrons can only be in the top (bottom) edge for positive (negative)
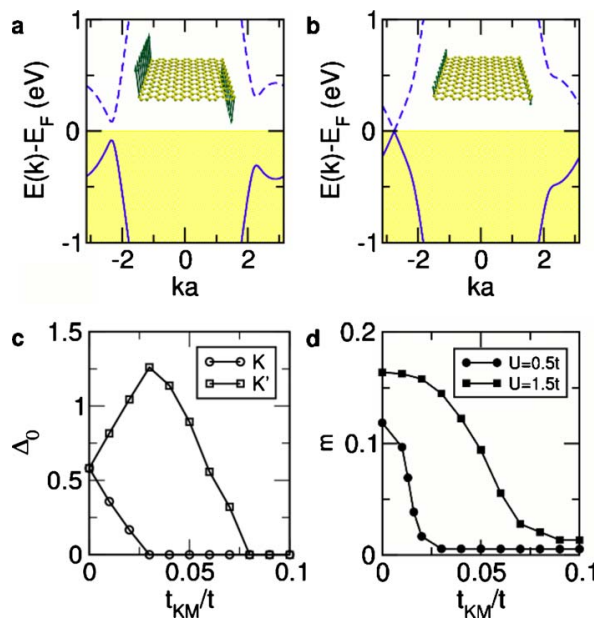

FIG. 2. (Color online) Valence and conduction band of a $N_{y}$ $=12$ ribbon with $U=t=3 \mathrm{eV}$ for two cases: (a) $\mathrm{AF}$ insulator with $t_{K M}=0.01 t$, (b) AF valley half metal $t_{K M}=0.03 t$. Insets: calculated magnetic density along the ribbon cell. (c) Evolution of the gaps $\Delta_{0}(K)$ and $\Delta\left(K^{\prime}\right)$ as a function of the SO coupling. (d) Depletion of the edge magnetic moment as the SO coupling $t_{K M}$ is increased for two different values of $U / t$.

velocity states. Spin $\uparrow$ and $\downarrow$ electrons can also be in the bottom edge but with velocity opposite to that of the top edge. Thus, the ribbon is conducting, the edges are not spinpolarized but the states at the Fermi energy carry a net spin current. The electronic structure of the same ribbon, but now taking $t_{K M}=0, U>0$, is radically different. Instead of the linear spin-filtered bands there is a gap, $\Delta_{0}$ so that the system is insulating. The wave functions of the valence band are shown in Fig. 1(e). They do not show correlation between spin and velocity but they are edge-sensitive: top (bottom) edge is ferromagnetic with spin-up (down) majority. The solution with reversed spins is equally valid.

In the light of Fig. 1, the question of how these competing electronic phases merge when both the Coulomb repulsion and the SO coupling are present calls for an answer. In Fig. 2 we show the energy bands for two cases, both with $N_{y}=12$, $U=t=3 \mathrm{eV}$, suitable for graphene (see supplementary material $^{27}$ ) and counterpolarized ferromagnetic edges. In Fig. 2(a) or Fig. 2(b) we take $t_{K M}=0.01 t\left(t_{K M}=0.03 t\right)$. As shown in the inset, the magnetic moments are localized in the edges. In the small $t_{K M}$ case, the system in an insulator, but the interedge gaps $\Delta_{0}$ are now valley dependent. In the large $t_{K M}$ case, the gap in one of the valleys closes completely, yet the edges are magnetic. This phase is radically different from the SO free case: a valley half metal with magnetic edges antiferromagnetically (AF) oriented.

The evolution of the valley symmetry breaking, reflected by the different size of $\Delta_{0}(K)$ and $\Delta_{0}\left(K^{\prime}\right)$, is shown in Fig. 2(c). The valley symmetry breaking can be understood using perturbative arguments. Close to the Dirac point the KaneMele-Hamiltonian can be approximated ${ }^{3}$ by $V_{K M}$ $=3 \sqrt{3} t_{K M} \sigma_{z} \tau_{z} \lambda_{z}$, where $\sigma_{z}, \tau_{z}$, and $\lambda_{z}$ are the spin, valley, and sublattice index, respectively. Let us consider first the $\left(t_{K M}\right.$ $=0, U>0)$ antiferromagnetic phase [Figs. 1(c) and 1(e)] as starting point. The valence band is made of states with $\sigma_{z}$ $=\uparrow$ and sublattice $\lambda_{z}=+$ (bottom edge) and states with $\sigma_{z}$ 

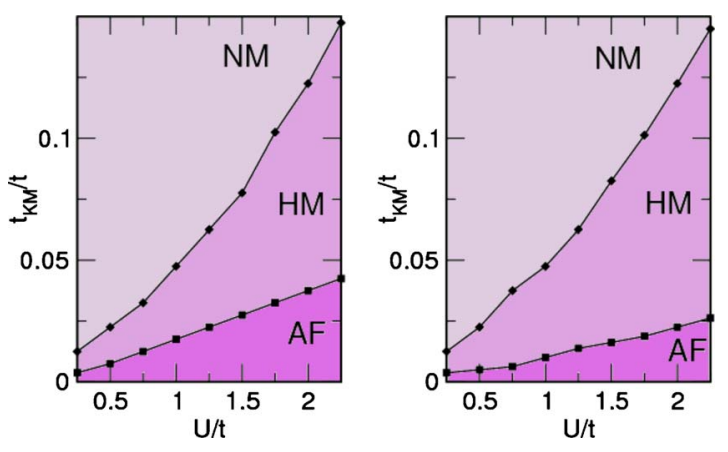

FIG. 3. (Color online) Phase diagrams for the AF insulating, AF valley $\mathrm{HM}$, and nonmagnetic (NM) phases for the ribbon with $N_{y}$ $=12$ (left) and $N_{y}=24$ (right).

$=\downarrow$ and sublattice $\lambda_{z}=-$ (upper edge) so that in both cases the product $\sigma_{z} \lambda_{z}$ has the same sign. Now it is apparent that the expectation value of $\sigma_{z} \tau_{z} \lambda_{z}$ has opposite signs in opposite valleys so that in one case the gap opens and in the other closes, as shown in Fig. 2(a).

In Fig. 2(d) we show how the magnetic moment in the edge atoms, $m=\frac{\left\langle n_{\uparrow}\right\rangle-\left\langle n_{\downarrow}\right\rangle}{2}$ is depleted as the strength of the spin-orbit coupling is increased, reflecting the competition between the two terms in the Hamiltonian. Above a certain value of $t_{K M}$, the magnetic moment vanishes altogether and a spin Hall insulator phase with conducting edge states identical to that with $U=0$ is obtained. The calculated phase diagram in the $\left(t_{K M}, U\right)$ plane showing the three different phases with zero total spin is shown in Fig. 3(a) or Fig. 3(b), for the case of $N_{Y}=12\left(N_{Y}=24\right)$. The phase diagram does not depend dramatically on the ribbon width. For freestanding intrinsic graphene $t_{K M}<10^{-4} t$ so that the ribbon would be in the insulating phase with magnetic edges.

In the noninteracting Kane-Mele model a nonequilibrium current $I$ induces spin accumulation $m$ in the edges. ${ }^{3}$ This can be quantified as follows. We assume that a population of nonequilibrium extra electrons occupies the positive velocity states so that the Fermi energy is increased by $\delta E_{F}=e V$. Using Landauer formula we have $I=2 \frac{e}{h} \delta E_{F}$, half of which goes on the top edge. From the noninteracting conduction-band dispersion $\epsilon_{k}=\hbar v_{F} k$, we obtain $\delta k_{F}$ and the corresponding change in density due to $\delta E_{F}, \delta n=\frac{\delta q}{a}=\frac{1}{\pi} \delta k_{F}$. Since $\delta E_{F}$ is small, this extra density goes as spin $\uparrow$ to one edge and spin $\downarrow$ to the other. Thus, half of the extra charge goes to each edge fully spin polarized so that the edge magnetic moment reads $\delta m=\frac{1}{4} \delta q=\frac{1}{4 \pi} \delta k_{F} a$. We can write the current in a given edge as

$$
I_{e d g e}=4 \pi \frac{e v_{F}}{a} m
$$

We now show that this picture survives in the interacting case in equilibrium. We find that in the ground state of the magnetically ordered topological insulator phases shown in Fig. 2, charge currents flow on the edges, the total current across a unit cell being null, in agreement with general theorems. ${ }^{28}$ The current operator is given by the sum of link currents associated with all the sites $b$ connected to $a$ by single-particle hopping,
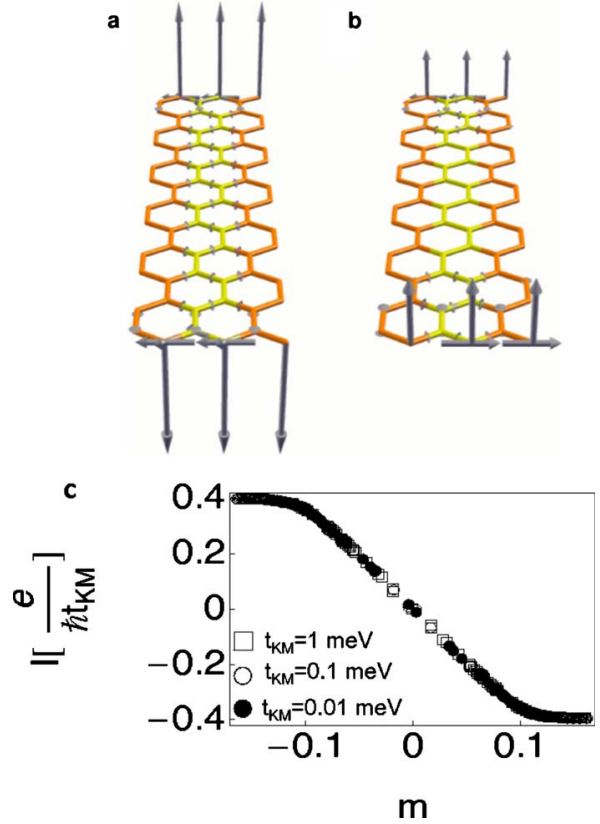

FIG. 4. (Color online) Current and magnetization maps for the $N_{y}=12$ ribbon with two solutions: (a) the counterpolarized insulating and (b) the copolarized and conducting, both with $t_{K M}=0.03 t$ and $U=t$. The current between two atoms $\vec{r}_{a}$ and $\vec{r}_{b}$ is plotted as a vector along the line $\vec{r}_{b}-\vec{r}_{a}$, starting in the midpoint. (c) Edge charge current, in units of $\frac{e}{\hbar} t_{K M}$, as a function of the magnetic moment of the edge, for three values of $t_{K M}=10^{-2}, 10^{-1}$, and $1 \mathrm{meV}$, for the AF phases.

$$
\hat{I}_{a}=\frac{e i}{\hbar}\left[\sum_{b \sigma} t_{a, b}(\sigma) c_{a, \sigma}^{\dagger} c_{b, \sigma}-t_{b, a}(\sigma) c_{b, \sigma}^{\dagger} c_{a, \sigma}\right] .
$$

For a given eigenstate $\Psi_{k \sigma \nu}$ of the mean-field Hamiltonian the current across the link $a b$ reads

$$
I_{a b}[\Psi]=2 \frac{e}{\hbar N} \operatorname{Im}\left[\Psi_{k \sigma \nu}^{*}(b) \Psi_{k, \sigma, \nu}(a) t_{a b}(\sigma) e^{i \phi_{a b}}\right]
$$

where $\phi_{a b}=0$ for $a$ and $b$ in the same cell, $\phi_{a b}= \pm k a$ if $a$ and $b$ are in adjacent cells, and $N$ is the number of cells in the crystal. The expectation value of this operator in the manybody ground state is obtained summing over all occupied bands: $\left\langle I_{a b}\right\rangle=\sum_{k, \nu, \sigma} f\left[\epsilon_{\nu \sigma}(k)\right] I_{a b}$. The average current is defined in the links of any pair of atoms connected by hopping in the one-body Hamiltonian. In Fig. 4 we plot the groundstate current map for the $N_{y}=12$ ribbon for the AF insulating phase [Fig. 2(a)] and the ferromagnetic conducting phase (supplementary material ${ }^{27}$ ). The AF valley half metal (not shown) is very similar to the AF insulator. The two electronic phases described in Fig. 2 as well as the ferromagnetic phase, present edge current of similar magnitude. Whereas in the FM case, currents flows in opposite directions in opposite edges, in the AF phases current runs parallel at the two edges. These results can be rationalized as if the magnetization plays the role of an external magnetic field. Thus, in the ferromagnetic case current flow is the same as in a quantum Hall bar. In the antiferromagnetic phases, though, current flows parallel at the two edges, as if a magnetic field was 
pointing along opposite directions at the two edges.

The magnitude of the top-edge current, normalized by $\frac{e}{\hbar} t_{K M}$, as a function of the top-edge magnetization $m$ collapses for several values of $t_{K M}$ [Fig. 4(c)]. For small $m$ the curve is linear, $I_{\text {edge }} \simeq-4 \frac{e}{\hbar} t_{K M} m$, in qualitative agreement with the analytical result of Eq. (2), since $v_{F}=\gamma t_{K M} a / \hbar$ with $\gamma \simeq 6.5$. At larger $m$ the edge current saturates to $\left|I_{\text {edge }}\right|$ $\simeq 0.4 \frac{e}{\hbar} t_{K M}$. If we take $t_{K M}=10 \mu \mathrm{eV}$, close to the small values obtained by $a b$ initio calculations for freestanding graphene, ${ }^{29}$ we obtain an edge current of $\simeq 0.4 \mathrm{nA}$, well within reach of state-of-the-art persistent current detection. ${ }^{30}$

In conclusion, we propose a mechanism for persistent charge currents. It occurs when Coulomb interaction gives rise to magnetic order in the edge states of the quantum spin Hall insulator model proposed by Kane and Mele., ${ }^{3,4}$ We study this scenario in graphene zigzag ribbons but it could occur in other systems as well. ${ }^{10-12}$ We find three new elec- tronic phases in that system that combine ferromagnetic order and spontaneous charge current flow, both localized in the zigzag edges. They arise from the interplay of Coulomb repulsion and spin-orbit coupling. When the ferromagnetic edges are counterpolarized, the valley symmetry is broken and, above a critical strength of the spin-orbit coupling, the system goes from an insulating to a valley-half-metal phase. In the three phases, current flows as if there was a real magnetic field perpendicular to the sample along the direction of the magnetization edge.

This work has been financially supported by MEC-Spain (Grants No. MAT07-67845 and CONSOLIDER No. CSD2007-0010). We are indebted to J. J. Palacios, D. Gosalbez Martinez, C. Untiedt, F. Guinea, L. Brey, and A. S. Nuñez, for fruitful discussions.
${ }^{1}$ P. W. Anderson, Basic Notions of Condensed Matter Physics (Benjamin/Cummings, Menlo Park, CA, 1983).

${ }^{2}$ M. Hasan and C. Kane, arXiv:1002.3895 (unpublished); J. E. Moore, Nature (London) 464, 194 (2010).

${ }^{3}$ C. L. Kane and E. J. Mele, Phys. Rev. Lett. 95, 226801 (2005).

${ }^{4}$ C. L. Kane and E. J. Mele, Phys. Rev. Lett. 95, 146802 (2005).

${ }^{5}$ B. A. Bernevig, T. L. Hughes, and S. C. Zhang, Science 314, 1757 (2006); X. L. Qi, Y. S. Wu, and S. C. Zhang, Phys. Rev. B 74, 085308, (2006); D. N. Sheng, Z. Y. Weng, L. Sheng, and F. D. M. Haldane, Phys. Rev. Lett. 97, 036808 (2006).

${ }^{6}$ N. A. Sinitsyn, J. E. Hill, H. Min, J. Sinova, and A. H. MacDonald, Phys. Rev. Lett. 97, 106804 (2006).

${ }^{7}$ M. Konig et al., Science 318, 766 (2007).

${ }^{8}$ Y. L. Chen et al., Science 325, 178 (2009).

${ }^{9}$ F. D. M. Haldane, Phys. Rev. Lett. 61, 2015 (1988).

${ }^{10}$ K. L. Lee, B. Gremaud, R. Han, B. G. Englert, and C. Miniatura, Phys. Rev. A 80, 043411 (2009).

${ }^{11}$ M. Gibertini, A. Singha, V. Pellegrini, M. Polini, G. Vignale, A. Pinczuk, L. N. Pfeiffer, and K. W. West, Phys. Rev. B 79, 241406 (2009).

${ }^{12}$ A. Shitade, H. Katsura, J. Kuneš, X. L. Qi, S. C. Zhang, and N. Nagaosa, Phys. Rev. Lett. 102, 256403 (2009).

${ }^{13}$ K. Nakada, M. Fujita, G. Dresselhaus, and M. S. Dresselhaus, Phys. Rev. B 54, 17954 (1996).

${ }^{14}$ M. Fujita, K. Wakabayashi, K. Nakada, and K. Kusakabe, J. Phys. Soc. Jpn. 65, 1920 (1996).

${ }^{15}$ L. Brey and H. A. Fertig, Phys. Rev. B 73, 235411 (2006).

${ }^{16}$ Y.-W. Son, M. L. Cohen, and S. G. Louie, Nature (London) 444,
347 (2006).

${ }^{17}$ A. H. Castro Neto, F. Guinea, N. M. R. Peres, K. S. Novoselov, and A. K. Geim, Rev. Mod. Phys. 81, 109 (2009).

${ }^{18}$ A. Rycerz, J. Tworzydo, and C. W. J. Beenakker, Nat. Phys. 3, 172 (2007).

${ }^{19}$ D. Gunlycke et al., Nano Lett. 7, 3608 (2007).

${ }^{20}$ O. V. Yazyev and M. I. Katsnelson, Phys. Rev. Lett. 100, 047209 (2008).

${ }^{21}$ X. Li, X. Wang, L. Zhang, S. Lee, and H. Dai, Science 319, 1229 (2008).

${ }^{22}$ J. Fernández-Rossier, Phys. Rev. B 77, 075430 (2008).

${ }^{23}$ W. Y. Kim and K. S. Kim, Nat. Nanotechnol. 3, 408 (2008).

${ }^{24}$ F. Muñoz-Rojas, J. Fernández-Rossier, and J. J. Palacios, Phys. Rev. Lett. 102, 136810 (2009).

${ }^{25}$ H. Min, J. E. Hill, N. A. Sinitsyn, B. R. Sahu, L. Kleinman, and A. H. MacDonald, Phys. Rev. B 74, 165310 (2006).

${ }^{26}$ Y. Yao, F. Ye, X. L. Qi, S. C. Zhang, Z. Fang, Phys. Rev. B 75, 041401(R) (2007).

${ }^{27}$ See supplementary material at http://link.aps.org/supplemental/ 10.1103/PhysRevB.82.161302 for a detailed comparison of the electronic structure and atomic magnetic moments obtained using the mean-field Hubbard approximation for the zigzag ribbon used throughout this report with those obtained using first principles.

${ }^{28}$ D. Bohm, Phys. Rev. 75, 502 (1949).

${ }^{29}$ M. Gmitra, S. Konschuh, C. Ertler, C. Ambrosch-Draxl, and J. Fabian, Phys. Rev. B 80, 235431 (2009).

${ }^{30}$ A. C. Bleszynski-Jayich et al., Science 326, 272 (2009). 